\title{
ON MULTIPLE BORSUK NUMBERS IN NORMED SPACES
}

\author{
ZS. LÁNGI ${ }^{1}$ and M. NASZÓDI ${ }^{2}$ \\ ${ }^{1}$ Author: Please provide affiliation and mailing address \\ e-mail: and email address \\ 2 Author: Please provide affiliation and mailing address \\ e-mail: and email address \\ Communicated by G. Fejes Tóth
}

(Received April 1, 2014; accepted December 12, 2015)

\begin{abstract}
Hujter and Lángi defined the $k$-fold Borsuk number of a set $S$ in Euclidean $n$-space of diameter $d>0$ as the smallest cardinality of a family $\mathcal{F}$ of subsets of $S$, of diameters strictly less than $d$, such that every point of $S$ belongs to at least $k$ members of $\mathcal{F}$.

We investigate whether a $k$-fold Borsuk covering of a set $S$ in a finite dimensional real normed space can be extended to a completion of $S$. Furthermore, we determine the $k$-fold Borsuk number of sets in not angled normed planes, and give a partial characterization for sets in angled planes.
\end{abstract}

\section{Introduction}

In 1933, Borsuk [5] posed the problem whether any set $S$ of diameter $d>0$ in Euclidean $n$-space $\mathbb{R}^{n}$ is the union of $n+1$ sets of diameters less than $d$. A proof of the affirmative answer for $n=2$ appeared in [5], and for $n=3$ in [6] (for finite $S$, see [9], [11]). Sixty years after the problem appeared, Kahn and Kalai [14] proved that for large values of $n$ the answer is negative. For surveys on Borsuk's problem, see [3,18].

Boltyanski [1] gave a characterization of bounded sets according to their Borsuk number (that is, the least number of smaller diameter pieces that they can be partitioned into) in the Euclidean plane: Let $\emptyset \neq S \subset \mathbb{R}^{2}$ be $a$

2010 Mathematics Subject Classification. Primary 52C17, 05C15, 52A21.

Key words and phrases. Borsuk's problem, diameter, unique completion, covering, bodies of constant width, complete, multiple chromatic number.. 
bounded set that is not a singleton. Then the Borsuk number of $S$ is 3 if $S$ has a unique completion (see Definition 2.1) and 2 otherwise.

Grünbaum [8] was the first to consider the Borsuk numbers of sets with respect to a metric distinct from the Euclidean, and determined the Borsuk numbers of sets in the plane equipped with the $\ell_{\infty}$ norm. The problem was solved for arbitrary normed planes in [4]:

THEOREM 1.1 (Boltyanski-Soltan). Let $S$ be a compact set in the normed plane with unit ball $\mathbf{B}$. Then the Borsuk number of $S$ is

- $a(S)=4$ if, and only if, $\mathbf{B}$ and $S$ are homothetic parallelograms;

- $a(S)=3$ if, and only if, $a(S) \neq 4$, there is a unique completion $C$ of $S$ with respect to $\mathbf{B}$, and $S$ satisfies the supporting line property: for any pair of parallel supporting lines of $C, S$ has a point on at least one;

- $a(S)=2$ otherwise.

As a generalization of Borsuk's problem, Hujter and Lángi [12] defined the $k$-fold Borsuk number, $a_{k}(S)$, of a set $S$ of diameter $d>0$ as the smallest cardinality of a family $\mathcal{F}$ of subsets of $S$, of diameter strictly less than $d$, such that every point of $S$ belongs to at least $k$ members of $\mathcal{F}$. Among other results, they determined the $k$-fold Borsuk numbers of any set in the Euclidean plane.

Motivated by Boltyanski's result, we investigate whether a ( $k$-fold) Borsuk covering of a set $S$ can be extended to a completion of $S$. Theorem 3.2 states that such an extension is possible in certain Minkowski spaces (ie. finite dimensional real normed spaces) provided that $S$ has a unique completion. The class of these Minkowski spaces include Euclidean $n$-space for all $n$. This result has been known in the Euclidean plane [1] but is new in higher dimensional Euclidean spaces. In Theorem 3.4, we extend this result to not angled Minkowski planes (see Definition 3.3).

In Theorems 4.5, 4.6 and 4.7, we find the $k$-fold Borsuk numbers of sets in not angled normed planes, and of sets that cannot be completed uniquely to a Reuleaux polygon in angled planes.

\section{Definitions and notation}

We denote the closed unit ball centered at a point $x \in \mathbb{R}^{n}$ of a Minkowski space by $\mathbf{B}(x)$, and its boundary, the unit sphere by $\mathbb{S}(x)$. For a set $A$, the intersection of unit balls centered at the points of $A$ is denoted as

$$
\mathbf{B} A=\bigcap_{x \in A} \mathbf{B}(x) .
$$


Definition 2.1. A bounded set $C$ in an n-dimensional Minkowski space is complete, if no set of the same diameter properly contains $C$. (Note that a complete set is clearly compact and convex.) $A$ set $S$ is a set of unique completion if there is a unique complete set $C$ containing $S$ of the same diameter as $S$.

Proposition 2.2. Let $S$ be a set of unit diameter in an $n$-dimensional Minkowski space. Then

- $S$ is complete if, and only if $S=\mathbf{B} S$,

- $S$ is a set of unique completion if, and only if, $\mathbf{B} S=\mathbf{B}^{2} S$ ie. $\mathbf{B} S$ is complete.

The first statement is due to Eggleston [7], where it is called the spherical intersection property, the second is due to Moreno (Corollary 3 in [16]). Note that in the second case the completion of $S$ is $\mathbf{B} S$.

We define the distance of a set $A$ of a Minkowski space and a point $x$ as $d_{\mathbf{B}}(x, A)=\inf \left\{d_{\mathbf{B}}(x, a): a \in A\right\}$, where $d_{\mathbf{B}}(x, a)$ is the distance of the points $a$ and $x$ in the normed space with unit ball $\mathbf{B}$.

\section{Extending a Borsuk covering in certain Minkowski spaces}

Our goal is to extend a Borsuk covering of a closed set $S$ of unique completion in a Minkowski space to its unique completion $\mathbf{B} S$. In general, a Borsuk covering of a compact set may not extend to any of its completions: consider a pair of points which in Euclidean space have many completions, all of whose Borsuk number is above two.

\subsection{Extension of a Borsuk covering in certain Minkowski spaces}

We define the following "Lens Cutting Condition" which holds in certain Minkowski spaces:

For any two distinct points $u$ and $v$ in $\mathbb{R}^{n}$ with $d_{\mathbf{B}}(u, v) \leq 1$ and $x \in \mathbb{S}(u) \cap \mathbb{S}(v)$ and $\varepsilon>0$, there is a $w \in \mathbb{R}^{n}$ such that $x \notin \mathbf{B}(w)$ but $\mathbf{B}(w) \supset \mathbf{B}(u) \cap \mathbf{B}(w) \backslash \varepsilon \mathbf{B}(x)$.

REMARK 3.1. It is not hard to see that (LCC) holds in all Euclidean spaces.

THEOREM 3.2. If (LCC) holds in a Minkowski space then any $k$-fold Borsuk covering of a closed set of unique completion extends to a $k$-fold Borsuk covering of its completion.

Proof. We prove the Theorem for $k=1$, the general case follows from the same argument. Let $S=Q_{1} \cup \ldots \cup Q_{k}$ be a Borsuk covering of a closed 
set $S$ of unique completion by closed sets of diameter at most $r<1$. Note that a Borsuk covering of the boundary of a set may be extended to the set in a straightforward way (cf. also Remark 4.3). Thus, we will define sets $Q_{1}^{\prime} \cup \ldots \cup Q_{m}^{\prime}=\operatorname{bd} \mathbf{B} S$ that form a Borsuk covering of the boundary of the completion $\mathbf{B} S$ of $S$.

For all $i, Q_{i}^{\prime}$ will contain $Q_{i} \cap \mathrm{bd} \mathbf{B} S$ and some more points of bd $\mathbf{B} S$. For an $x \in(\mathrm{bd} \mathbf{B} S)$ we take the index $i$ such that $d\left(x, Q_{i}\right)$ is minimal (if it is not unique, we take all such $i$ ), and include $x$ into $Q_{i}^{\prime}$. Clearly, $Q_{i}^{\prime}$ is closed.

Note that for any $x \in \mathbf{B} S \backslash S$ we have that

$$
\begin{aligned}
& \text { there are no two distinct points } u, v \in \mathbf{B} S \text { with } d_{\mathbf{B}}(x, u)= \\
& d_{\mathbf{B}}(x, v)=1 \text {. }
\end{aligned}
$$

Suppose the contrary. Then $S \subseteq \mathbf{B}^{2} S \subseteq \mathbf{B}(u) \cap \mathbf{B}(v)$. On the other hand, $\mathbf{B}^{2} S$ is the intersection of all unit balls that contain $S$, and hence by (LCC), $\mathbf{B}^{2} S \subseteq(\mathbf{B}(u) \cap \mathbf{B}(v)) \backslash\{x\}$, contradicting $x \in \mathbf{B} S=\mathbf{B}^{2} S$.

The family of the sets $q_{i}^{\prime}$ is a Borsuk partition of bd $\mathbf{B} S$. Indeed, let $x, y \in Q_{i}^{\prime}$. If $x$ or $y$ is in $S$ then clearly, $d(x, y)<1$. If both are in $Q_{i}^{\prime} \backslash S$ then, by $\left(^{*}\right), d(x, y)<1$.

\subsection{Extension of a Borsuk covering in certain Minkowski planes}

It is not difficult to see that a strictly convex normed plane (that is, when the unit disk B is strictly convex) satisfies (LCC), and thus has the extension property of Theorem 3.2. Next, we consider a class of Minkowski planes that is wider than the class of strictly convex planes, and where (LCC) does not hold, but the extension property still does. The following definition is from [3] (cf. also [4]).

DeFinition 3.3. A normed plane with unit ball $\mathbf{B}$ is angled, if for some non-collinear points $a, b, c$, we have $[a, b] \cup[b, c] \subset \mathbb{S}$.

THEOREM 3.4. Let $S$ be a set of unique completion in a not angled normed plane, and let $C$ be the completion of $S$. Then any $k$-fold Borsuk covering $\mathcal{F}$ of $S$ can be extended to a $k$-fold Borsuk covering of $C$.

From this point on throughout this section, we assume that the Minkowski plane we work with is not angled.

The following monotonicity lemma appeared in [15].

Lemma 3.5 (Lassak). Let $t \mapsto p(t)$ (with $t \in[0,1]$ ) be a simple, closed, continuous curve, defining the boundary of a complete body of diameter one in a Minkowski plane. Let $p=p(0)$, and let $t_{1}$ and $t_{2}$ be the smallest and the largest values of $t$ such that $\operatorname{dist}_{\mathbf{B}}(p, p(t))=2$. Then the function $t \mapsto$ $\operatorname{dist}_{\mathbf{B}}(p, p(t))$ is

- strictly increasing on the interval $\left[0, t_{1}\right]$,

- equal to one on $\left[t_{1}, t_{2}\right]$, and 
- strictly decreasing on $\left[t_{2}, 1\right]$.

Corollary 3.6. Let $C$ be a complete body of diameter one in a Minkowski plane. Then, for any $p \in \operatorname{bd} C$ we have the following.

- The set of points of $C$ at unit distance from $p$ is a connected arc of $\mathbb{S}(p) \cap \operatorname{bd} C$.

- If $\|q-p\|_{\mathbf{B}}=\|r-p\|_{\mathbf{B}}$ for some $q, r \in \operatorname{bd} C$, then the arc of bd $C$, connecting $q$ and $r$ and not containing $p$, belongs to the circle $\mathbb{S}(p)$.

LEMma 3.7. If $C$ is a complete body in a Minkowski plane, and $[a, b]$, $[c, d]$ are two disjoint diameters of $C$ such that $a, b, c, d$ are in counterclockwise order in bd $C$, then $[a, d],[b, c] \subset$ bd $C$ and they are parallel.

Proof. Consider the quadrangle $Q=\operatorname{conv}\{a, b, c, d\}$. Observe that as $[a, b]$ and $[c, d]$ are diameters of $C$, neither $C$ nor $Q \subseteq C$ contains a translate of neither $[a, b]$ nor $[c, d]$ in its interior. Thus, $[a, c]$ and $[b, d]$ are parallel, and they belong to bd $C$.

Lemma 3.8 is a straightforward consequence of Theorems 33.7 and 33.9 of $[3]$.

LEMMA 3.8. Let $S$ be a compact set of unique completion in a not angled normed plane, and let $C$ be its completion. Then, for any parallel supporting lines $L$ and $L^{\prime}$ of $C, L$ or $L^{\prime}$ contains a point of $S$. In other words, $S$ satisfies the supporting line property (see page 2).

Lemma 3.9. Let $S$ be a compact set of diameter one and of unique completion, $C$. Then, for any point $x \in(\mathrm{bd} C) \backslash S$, there is an open circle arc of radius one, containing $p$ and being contained in bd $C$, such that its endpoints and its center belong to $S$.

Proof. Let $x \in(\operatorname{bd} C) \backslash S$. Then, since $\mathbf{B} S=C$, there is a point $p \in S$ such that $x \in \mathbb{S}(p)$. Clearly, $[p, x]$ is a diameter of $C$, and thus, $p \in \operatorname{bd} C$. Let $L$ and $L^{\prime}$ be a pair of parallel supporting lines of $C$ such that $x \in L$ and $p \in L^{\prime}$. For simplicity, we imagine these lines as vertical such that $L$ is to the left of $L^{\prime}$. Let $[a, b]=C \cap L$ and $[c, d]=C \cap L^{\prime}$, and note that these segments might be degenerate. Without loss of generality, we assume that $a, b, c$ and $d$ are in this counterclockwise order in bd $C$.

First, we show that at least one of $a$ and $c$ belongs to $S$. Indeed, consider a sequence of supporting lines $L_{m}$ of $C$, with positive slopes, such that the limit of $L_{m} \cap C$ is $\{a\}$. For any $m$, let $L_{m}^{\prime}$ be the supporting line of $C$, parallel to and different from $L_{m}$. Clearly, the limit of $L_{m}^{\prime} \cap C$ is $\{c\}$. Now, by Lemma 3.8, we have that for any $m, L_{m}$ or $L_{m}^{\prime}$ contains a point of $S$. Thus, the observation follows from the compactness of $S$. We may show similarly that at least one of $b$ and $d$ belongs to $S$.

Now we prove the assertion. If both $[a, x]$ and $[x, b]$ contain a point of $S$, then we may observe that $[a, b] \subset \mathbb{S}(p)$, and thus, our lemma follows. Assume that exactly one of these segments, say $[x, b]$, contains a point of $S$. Then 
$a \notin S$, and thus, $c \in S$. Let $G$ be the arc of $(\operatorname{bd} C) \cap \mathbb{S}(c)$, starting at $x$ and above the line connecting $x$ and $c$. If $G$ does not contain a point of $S$, then for some point $c^{\prime} \in L^{\prime} \backslash[c, d]$, we have $S \subset \mathbf{B}\left(c^{\prime}\right)$; or in other words, $c^{\prime} \in \mathbf{B} S=C$; a contradiction. Thus, $G$ contains a point of $S$, which yields the assertion.

We are left with the case that $[a, b] \cap S=\emptyset$, which, in particular, implies that $c, d \in S$. Note that if $c \neq d$, then, by Lemma 3.7, for any $y \in \operatorname{relint}[c, d]$, we have $C \cap \mathbb{S}(y)=[a, b]$. Thus, moving $y$ slightly to the right, we can find a point $y^{\prime}$ such that $S \subset \mathbf{B}\left(y^{\prime}\right)$, but $[a, b] \cap \mathbf{B}\left(y^{\prime}\right)=\emptyset$. This yields that $C \not \subset \mathbf{B}\left(y^{\prime}\right)$, or in other words that $y^{\prime} \notin \mathbf{B} C=C$, contradicting $y^{\prime} \in \mathbf{B} S=C$. Thus, we obtain that $c=d$. In this case, similarly like in the previous paragraph, one can show that both $\operatorname{arcs}$ of $(\mathrm{bd} C) \cap \mathbf{B}(c)$, starting at $x$, contain a point of $S$, and the assertion readily follows.

Theorem 3.4. Note that it suffices to extend $\mathcal{F}$ to a $k$-fold Borsuk covering of bd $C$.

Let $\mathcal{F}=\left\{Q_{1}, Q_{2}, \ldots, Q_{m}\right\}$ be a $k$-fold Borsuk covering of $S$. Without loss of generality, we may assume that $S$ is compact. Let $\varepsilon$ be chosen in such a way that the diameter of every member of $\mathcal{F}$ is at most $1-3 \varepsilon$. Now, for every $i$, we set $Q_{i}^{*}=Q_{i}+\varepsilon B$, and observe that $\mathcal{F}^{*}=\left\{Q_{1}^{*}, Q_{2}^{*}, \ldots, Q_{m}^{*}\right\}$ is still a $k$-fold Borsuk covering of $S$.

Consider the connected components of bd $C \backslash S$. By Lemma 3.9, they are open circle arcs of unit radius, with their centers contained in $S$. Note that $\mathcal{F}^{*}$ is a $k$-fold covering of any such arc not longer than $2 \varepsilon$. Since bd $C$ has a bounded length, there are only finitely many arcs that are not covered $k$ fold by $\mathcal{F}^{*}$. Thus, by induction, it suffices to prove that $\mathcal{F}^{*}$ can be extended to cover $k$-fold at least one such arc.

Consider an arc $G$ that is not covered by $\mathcal{F}^{*} k$-fold. Let $p \in S$ denote the center, and $q, r \in S$ denote the endpoints of $G$. If, for every $x \in G, p$ is the only point of $C$ at unit distance from $x$, then we can apply the argument in the proof of Theorem 3.2. Thus, assume that for some $x \in G$ and $p^{\prime} \in C$ with $p \neq p^{\prime}$, we have $x \in \mathbb{S}\left(p^{\prime}\right)$, where without loss of generality, we may assume that, say, $[p, r]$ and $\left[p^{\prime}, x\right]$ are disjoint. Note that since $[p, r]$ and $\left[p^{\prime}, x\right]$ are diameters of $C$, we have that $\left[p, p^{\prime}\right]$ and $[x, r]$ are parallel, and are contained in bd $C$.

Let $L$ and $L^{\prime}$ be the line containing $[r, x]$ and $\left[p, p^{\prime}\right]$, respectively. Observe that the points diametrically opposite to any point in the relative interior of $L \cap C$ are the points of $L^{\prime} \cap C$. Let $y$ be the endpoint of $L \cap$ bd $C$ closer to $x$ than to $r$. If $q \in L$, then we may add the segment $[q, x]$ to any $Q_{i}^{*}$ containing $q$, and $[x, r]$ to any $Q_{i}^{*}$ containing $r$. Thus, we may assume that $y$ is a point of $G$.

Consider the case that the points diametrically opposite to $y$ are only the points of $L^{\prime} \cap C$. Then we may add the segment $[y, r]$ to any $Q_{i}^{*}$ containing $r$. On the other hand, note that if some $u \in \operatorname{bd} C$ is diametrically opposite 
to any point of the arc between $y$ and $q$, then it is diametrically opposite to $q$ as well. Thus, we may add this arc to any $Q_{i}^{*}$ containing $q$.

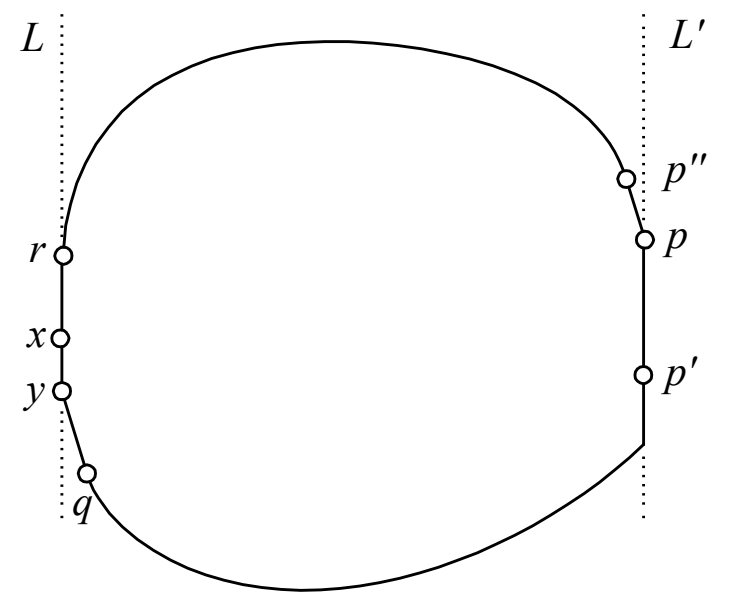

Fig. 1. An illustration for the proof of Theorem 3.4

Finally, assume that there is some point $p^{\prime \prime} \notin L^{\prime} \cap C$ that is diametrically opposite to $y$ (cf. Figure 1 ). Then, clearly, the points $p^{\prime}, p, p^{\prime \prime}, y$ are in this cyclic order in bd $C$, and $\left[y, p^{\prime \prime}\right]$ and $[q, p]$ are disjoint diameters of $C$, which yields, by Lemma 3.7, that $\left[p, p^{\prime \prime}\right]$ and $[q, y]$ are parallel, and both are contained in bd $C$. Thus, bd $C$, and also $\mathbb{S}(p)$, contains an angle, which contradicts the conditions of the theorem.

Corollary 3.10. Let $S$ be a set of unique completion in a not angled normed plane, and let $C$ be the completion of $S$. Then for any value of $k$, $a_{k}(S)=a_{k}(C)$.

\section{The multiple Borsuk numbers of sets in a not angled normed plane}

We start with three observations, which, for sets in a Euclidean space, appeared as Remarks 1-3 in [12]. Their proofs are straightforward modifications of those in [12], and hence we omit them.

REMARK 4.1. The sequence $a_{k}(S)$ is sub-additive for every set $S$ in any normed (or metric) space. More precisely, for any positive integers $k, l$, we have $a_{k+l}(S) \leq a_{k}(S)+a_{l}(S)$.

REMARK 4.2. Let $S$ be a set of diameter $d>0$ in a normed (or metric) space. Then for every set $S$ of diameter $d>0$ and every $k \geq 1$, we have 
$a_{k}(S) \geq 2 k$. Furthermore, for every value of $k$, if $a(S)=2$, then $a_{k}(S)=2 k$, and if $a(S)>2$, then $a_{k}(S)>2 k$.

REMARK 4.3. Let $S \subset \mathbb{R}^{n}$ be a set of positive diameter in a normed space. Then for every value of $k, a_{k}(S)=a_{k}(\mathrm{bd} S)$.

Let $S$ be a bounded set in a normed plane. By Theorem 1.1, if $S$ is not a set of unique completion then $a(S)=2$, which yields that for any $k$, $a_{k}(S)=2 k$. Combined with Corollary 3.10, it yields that it suffices to characterize the $k$-fold Borsuk numbers of complete sets. To do this, we need a generalization of the notion of Reuleaux polygons for normed planes (cf. also [21], [19] and [10]).

DeFinition 4.4. Let $C$ be a complete set in a normed plane. If $C$ is the intersection of finitely many translates of $\mathbf{B}$, we say that $C$ is a Reuleaux polygon. If $m$ is the smallest number such that $C$ is the intersection of $m$ translates of $\mathbf{B}$, then we say that $C$ has $m$ sides.

THEOREM 4.5. Let $C$ be a complete set of diameter one in a normed plane, which is not a Reuleaux polygon. Then for every $k, a_{k}(C)=2 k+1$.

Proof. Clearly, by Remark 4.2, for every $k$, we have $a_{k}(C) \geq 2 k+1$. Thus, we need to show that if $C$ is not a Reuleaux polygon, then $C$, or equivalently, bd $C$, can be covered $k$-fold by $2 k+1$ subsets of smaller diameters.

To do this, we prove the existence of $2 k+1$ diameters $\left[p_{i}, p_{2 k+1+i}\right]$, where $i=1,2, \ldots, 4 k+2$ of $C$, such that for any $j \neq 2 k+1+i,\left[p_{i}, p_{j}\right]$ is not a diameter of $C$. Observe that from this, the assertion follows. Indeed, by Lemma 3.5, we have that any two of these diameters intersect. Thus, we may label their endpoints in such a way that $p_{1}, p_{2}, \ldots, p_{4 k+2}$ are in counterclockwise order in $\mathrm{bd} C$. Let $A_{i}$ be the arc of bd $C$, connecting $p_{i}$ and $p_{2 k+i}$ and not containing $p_{2 k+1+i}$. Then $A_{i}$ is of diameter less than one, and the arcs $A_{1+k s}$, where $s=1,2, \ldots, 2 k+1$, form a $k$-fold Borsuk covering of bd $C$.

For simplicity, for any point $x \in \operatorname{bd} C$, we set $G(x)=C \cap \mathbb{S}(x) \subset$ bd $C$. We choose the required diameters as follows. Let $\left[p_{1}, p_{2 k+2}\right]$ be an arbitrary diameter of $C$. Let $q_{1}, r_{1}$ and $q_{2 k+2}, r_{2 k+2}$ be the endpoints of the arcs $G\left(p_{1}\right)$ and $G\left(p_{2 k+2}\right)$, respectively. It follows from Lemma 3.5 that $G\left(p_{2 k+2}\right) \subseteq G\left(q_{1}\right) \cup G\left(r_{1}\right)$ and $G\left(p_{1}\right) \subseteq G\left(q_{2 k+2}\right) \cup G\left(r_{2 k+2}\right)$. Then, as no finitely many unit circle arcs cover bd $C, X_{2}=\operatorname{bd} C \backslash\left(G\left(q_{1}\right) \cup G\left(r_{1}\right) \cup\right.$ $\left.G\left(q_{2 k+2}\right) \cup G\left(r_{2 k+2}\right)\right) \neq \emptyset$.

Observe that for any $x \in X_{2},\left\|x-p_{1}\right\|_{\mathbf{B}}$ and $\left\|x-p_{2 k+2}\right\|_{\mathbf{B}}$ are strictly less than one, and any point diametrically opposite to $x$ is also contained in $X_{2}$. Let $p_{2} \in X_{2}$ arbitrary. Since $C$ is complete, there is some $p_{2 k+3} \in$ bd $C$ such that $\left[p_{2}, p_{2 k+3}\right]$ is a diameter of $C$. Then $p_{2 k+3} \in X_{2}$; that is, $\left\|p_{2 k+3}-p_{1}\right\|_{\mathbf{B}}$ and $\left\|p_{2 k+3}-p_{2 k+2}\right\|_{\mathbf{B}}$ are strictly less than one. Let us define $q_{2}, r_{2}, q_{2 k+3}, r_{2 k+3}$ similarly as for $p_{1}$ and $p_{2 k+2}$. Now, set $X_{3}=X_{2} \backslash\left(G\left(q_{2}\right) \cup\right.$ $\left.G\left(r_{2}\right) \cup G\left(q_{2 k+3}\right) \cup G\left(r_{2 k+3}\right)\right)$. Since bd $C$ is not covered by finitely many 
unit circle arcs, we have $X_{3} \neq \emptyset$. Thus, using the argument as for $\left[p_{2}, p_{2 k+3}\right]$, we can find a diameter $\left[p_{3}, p_{2 k+4}\right]$ with $p_{3}, p_{2 k+4} \in X_{3}$, satisfying the required conditions. Since $C$ is not a Reuleaux polygon, repeating this procedure we may choose the required $2 k+1$ diameters for any value of $k$.

THeOREM 4.6. If $C$ is an m-sided Reuleaux polygon of diameter one in the not angled norm with unit disk $\mathbf{B}$, then

1. $m$ is an odd integer,

2. if $m=2 s+1$, then the $k$-fold Borsuk number of $C$ is $a_{k}(C)=2 k+\left\lceil\frac{k}{s}\right\rceil$.

Proof. Let $G_{i}$, where $i=1,2, \ldots, m$, be unit circle arcs that cover bd $C$, and let $p_{i}, q_{i}$ and $r_{i}$ be the center and the two endpoints of $G_{i}$, respectively. Clearly, we may assume that no $G_{i}$ is a proper subset of any unit circle arc in bd $C$.

We label the points in such a way that in counterclockwise order, $q_{i}$ is the starting and $r_{i}$ is the endpoint of $G_{i}$, and the points $q_{1}, q_{2}, \ldots, q_{m}$ are in this counterclockwise order in bd $C$. For simplicity, we call the $G_{i}$ s the sides, and their endpoints the vertices of $C$. Note that $r_{1}, r_{2}, \ldots, r_{m}$ are in this counterclockwise order as well, as otherwise $G_{i} \subset G_{j}$ for some $i \neq j$, which contradicts the assumption that $C$ is $m$-sided. By Lemma 3.5, we have that $p_{1}, p_{2}, \ldots, p_{m}$ are also in this counterclockwise order.

Since $C$ is complete, $p_{i} \in \operatorname{bd} C$ for every value of $i$. Furthermore, since $m$ is the minimal number of unit circle arcs that cover bd $C$, there is no point that belongs to more than two arcs. We observe also that if $p_{i}$ is in the relative interior of a segment $[x, y] \subset$ bd $C$, then, by Lemma 3.7, $G_{i}=\left[q_{i}, r_{i}\right]$ is a segment. Thus, replacing $G_{i}$ by, say $\mathbb{S}(x) \cap C$, we still have a family of $m$ unit circle arcs that cover bd $C$. This implies that, without loss of generality, we may assume that no $p_{i}$ is in the relative interior of a segment on $\operatorname{bd} C$.

Consider, first, the case that two consecutive sides, say $G_{i}$ and $G_{i+1}$ overlap. Then $q_{i}, q_{i+1}, r_{i}$ and $r_{i+1}$ are in this counterclockwise order in bd $C$. Thus, $\left[p_{i}, r_{i}\right]$ and $\left[p_{i+1}, q_{i+1}\right]$ are disjoint diameters, which yields, by Lemma 3.7, that $\left[p_{i}, p_{i+1}\right],\left[q_{i+1}, r_{i}\right] \subset$ bd $C$, and that they are parallel. Hence, for any two overlapping sides of $C$, the common part is a straight line segment.

Now we show that the intersection of any two consecutive sides of $C$ contains the center of exactly one side. Consider the sides $G_{i}$ and $G_{i+1}$.

Case 1, $G_{i}$ and $G_{i+1}$ do not overlap. Then $r_{i}=q_{i+1}$. Observe that $p_{i}, p_{i+1} \in \mathbb{S}\left(r_{i}\right) \cap C$. Let $G$ be the arc of bd $C$ connecting $p_{i}$ and $p_{i+1}$ and not containing $r_{i}$. We show that there is a point in the relative interior of $G$ which is diametrically opposite only to $r_{i}$. Note that since $C$ is a Reuleaux polygon, it yields that in this case $C \cap \mathbb{S}\left(r_{i}\right)$ must be a side of $C$.

Let $p$ be an arbitrary relative interior point of $G$, and assume that $C \cap$ $\mathbb{S}(p)$ contains not only $r_{i}$, but some other point $x$ as well. Without loss of generality, we may assume that $x \in G_{i}$, which yields that $\left[p_{i}, r_{i}\right]$ and $[p, x]$ are disjoint diameters of $C$. Thus, by Lemma $3.7,\left[p, p_{i}\right],\left[r_{i}, x\right] \subset$ bd $C$, and 
they are parallel. Since here $p$ is an arbitrary relative interior point of $G$, we have that either $G=\left[p_{i}, p_{i+1}\right]$ or there is some relative interior point $z$ of $G$ such that $G=\left[p_{i}, z\right] \cup\left[z, p_{i+1}\right]$. Observe that $G \subset C \cap \mathbb{S}\left(r_{i}\right)$, and hence, as B is not angled, it follows that $G=\left[p_{i}, p_{i+1}\right]$. Furthermore, for some point $x \in C$, we have that $\left[r_{i}, x\right] \subset$ bd $C$, and that $\left[r_{i}, x\right]$ and $\left[p_{i}, p_{i+1}\right]$ are parallel. This means that $\left[r_{i}, x\right]$ belongs to both $\mathbb{S}\left(p_{i}\right)$ and $\mathbb{S}\left(p_{i+1}\right)$, which contradicts our assumption that $G_{i}$ and $G_{i+1}$ do not overlap.

Case 2, $G_{i}$ and $G_{i+1}$ overlap; or in other words, $r_{i} \neq q_{i+1}$. Then, similarly like in Case 1 , we have that $\left[r_{i}, q_{i+1}\right],\left[p_{i}, p_{i+1}\right] \subset$ bd $C$, and they are parallel. Let $L$ and $L^{\prime}$ denote the line containing $\left[p_{i}, p_{i+1}\right]$ and $\left[r_{i}, q_{i+1}\right]$, respectively. Observe that $\mathbb{S}\left(p_{i}\right)$ and $\mathbb{S}\left(p_{i+1}\right)$ both contain $C \cap L^{\prime}$, and thus, we have $C \cap L^{\prime}=\left[r_{i}, q_{i+1}\right]$. Furthermore, note that, for any point $p$ in the relative interior of $\left[p_{i}, p_{i+1}\right]$, the points of $C$ diametrically opposite to $p$ are exactly the points of $\left[r_{i}, q_{i+1}\right]$. Thus, the center of any side of $C$ containing $p$ is a point of $\left[q_{i+1}, r_{i}\right]$. Since we chose the sides of $C$ in such a way that no center is contained in a straight line segment in bd $C$, we have that only $q_{i+1}$ or $r_{i}$ can be the center of a side, and also that $L \cap C=\left[p_{i}, p_{i+1}\right]$.

Suppose, for contradiction, that both $q_{i+1}$ and $r_{i}$ are centers, and let these sides be $G_{j}$ and $G_{j+1}$. Then, we have $\left[p_{i}, p_{i+1}\right] \subseteq G_{j} \cap G_{j+1}$, and, similarly like in the previous paragraph, we may obtain that $\left[p_{i}, p_{i+1}\right]=$ $G_{j} \cap G_{j+1}$. Thus, $q_{j+1}=p_{i}$ and $r_{j}=p_{i+1}$. Since $q_{i} \neq q_{i+1}=p_{j}$ and $q_{j} \neq$ $q_{j+1}=p_{i}$, it follows that $\left[q_{i+1}, q_{j}\right]$ and $\left[p_{i}, q_{i}\right]$ are disjoint diameters of $C$. Hence, by Lemma 3.7 , we have that $\left[q_{i}, q_{i+1}\right]$ and $\left[q_{j}, p_{i}\right]$ are parallel and contained in bd $C$. Thus, $\left[q_{i}, q_{i+1}\right]$ and $\left[q_{i+1}, r_{i}\right]$ are both contained in $\mathbb{S}\left(p_{i}\right)$, which contradicts our assumption that the normed plane is not angled.

We have shown that the intersection of any two consecutive sides contains the center of exactly one side. Since any point of bd $C$ belongs to at most two sides of $C$, these intersections are pairwise disjoint. As the number of centers is equal to the number of intersections, it follows that the center of every side of $C$ is contained in one of these intersections. In fact, we showed a bit more: every center is the vertex of some other side.

For every value of $i$, consider a point $z_{i}$ that belongs to $G_{i}$ but no other side of $C$. Note that since no point of bd $C$ belongs to more than two sides of $C$, this is possible, and also that, by Lemma 3.7, the segments $\left[p_{i}, z_{i}\right]$, where $i=1,2, \ldots, m$, are pairwise intersecting diameters of $C$. Clearly, the $2 m$ points $p_{i}$ and $z_{j}$ form an alternating sequence $S$ in bd $C$, and each of the two open arcs of bd $C$, starting at, say, $p_{1}$ and ending at $z_{1}$, contains exactly $m-1$ points. Since the subsequence of $S$ in any of the above two arcs, starts with some $z_{i}$ and ends with some $p_{j}$, we have that $m-1$ is an even number, and thus, $m$ is odd.

Now we prove the second part. Let $m=2 s+1$. According to the previous paragraph, we have that for every $i, p_{i} \in G_{i+s} \cap G_{i+s+1}$. First, we show that the points $z_{i}$ can be chosen in such a way that the set $Z=\left\{z_{i}: i=1,2, \ldots, m\right\}$ contains no diametrically opposite pair. 
Assume that for every $i, z_{i}$ belongs to only $G_{i}$, but $Z$ contains a diametrically opposite pair, say $z_{i}$ and $z_{j}$. Then $j=i-s$ or $j=i+s$. Without loss of generality, we may assume that $z_{i}$ and $z_{i+s}$ are diametrically opposite. From this, by Lemma 3.7, we obtain that $\left[z_{i}, p_{i+s}\right]$ and $\left[z_{i+s}, p_{i}\right]$ are parallel and are contained in bd $C$. Let $L$ be the line containing $\left[p_{i+s}, z_{i}\right]$. Note that $p_{i+s}$ is an endpoint of $L \cap C$, and let $x$ be the other endpoint. Observe that $p_{i-s} \notin L$, as otherwise $z_{i+s} \in G_{i-s}$, which is a contradiction. In addition, $x$ is not diametrically opposite to $z_{i-s}$. Indeed, if $\left[x, z_{i-s}\right]$ is a diameter, then $\left[x, z_{i-s}\right]$ and $\left[p_{i-s}, p_{i}\right]$ are disjoint diameters, and thus Lemma 3.7 yields that $\left[x, p_{i-s}\right],\left[z_{i-s}, p_{i}\right] \subset$ bd $C$, which contradicts our assumption that the normed plane is not angled (cf. Figure ??). Now we choose any point $y \in(\operatorname{bd} C) \backslash L$ sufficiently close to $x$, and replace $z_{i}$ by $y$. Then, clearly, $y$ is diametrically opposite to neither $z_{i-s}$ nor $z_{i+s}$. Thus, to choose a subset $Z$ that does not contain diametrically opposite points, we start with any set and then, applying the argument of this paragraph, we may replace the points one by one to reduce the number of diametrically opposite points.

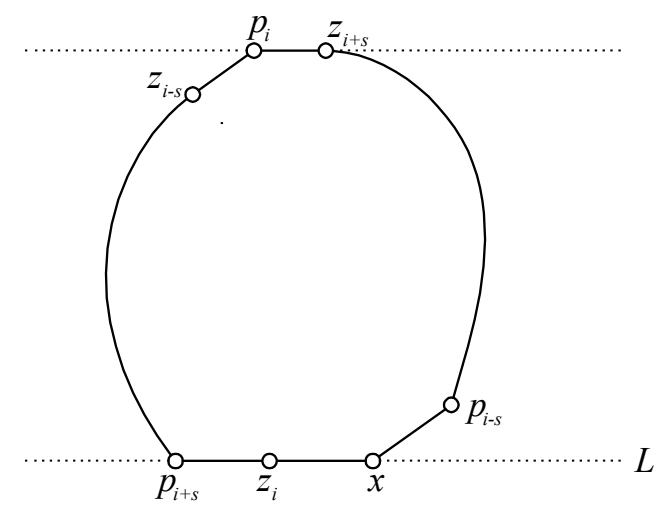

Fig. 2. An illustration for the proof of Theorem 4.6labelfig:Reuleaux

We constructed a subset $Z=\left\{z_{i}: i=1,2, m\right\}$ such that for every $i, z_{i}$ belongs only to $G_{i}$, and $Z$ contains no diametrically opposite pair. Let $A_{i}$ denote the closed arc of bd $C$, which, in counterclockwise order, starts at $z_{i}$ and ends at $z_{i+s}$. Observe that by Lemma 3.5 and the choice of $Z$, no such arc contains a diametrically opposite pair. On the other hand, the sets $A_{j s}$, where $j=0,1, \ldots, 2 k+\left\lceil\frac{k}{s}\right\rceil-1$ and the indices are taken mod $m$, covers bd $C k$-fold, and thus, they are a $k$-fold Borsuk covering of bd $C$. This proves that $a_{k}(C) \leq 2 k+\left\lceil\frac{k}{s}\right\rceil$.

To prove the other direction, we note that the $k$-fold Borsuk coverings of the set $\left\{p_{i}: i=1,2, \ldots, m\right\}$ can be identified with the $k$-fold vertex-colorings of a $(2 s+1)$-cycle. Since it is known (cf. [20]) that the $k$-fold chromatic number of such a cycle is $2 k+\left\lceil\frac{k}{s}\right\rceil$, the assertion follows. 
From Theorems 1.1, 4.5 and Remark 4.2, we immediately obtain the following.

TheOREM 4.7. Let $S$ be a set of positive diameter in a normed plane $\mathbf{B}$.

- If $S$ is not a set of unique completion, or $S$ does not satisfy the supporting line property, then for every value of $k, a_{k}(S)=2 k$.

- If $S$ is a set of unique completion that satisfies the supporting line property (see page 2) and the completion of $S$ is not a Reuleaux polygon, then for every $k, a_{k}(S)=2 k+1$.

\section{Remarks and Questions}

REMARK 5.1. We note that our results cannot be extended to angled planes. For example, Theorem 3.4 fails if the unit disk $\mathbf{B}$ is a parallelogram. Besides, any centrally symmetric polygon with $4 m$ sides is a Reuleaux polygon with $2 m$ sides in its norm (and thus, it has even sides according to our definition).

REMARK 5.2. The $k$-fold Borsuk number of an o-symmetric polygon $P$ with $2 m$ sides in its own norm is $a_{k}(P)=2 k+\left\lceil\frac{2 k}{m-1}\right\rceil$.

Proof. Let the vertices of the polygon be $p_{1}, p_{2}, \ldots, p_{2 m}$ in counterclockwise order. Then $p_{i}$ is diametrically opposite to $p_{i+k-1}, p_{i+k}$ and $p_{i+k+1}$. Thus, the inequality $a_{k}(P) \geq 2 k+\left\lceil\frac{2 k}{m-1}\right\rceil$ follows from the Pigeonhole Principle. On the other hand, if $G_{i}$ denotes the shorter arc in bd $P$, connecting the midpoints of $\left[p_{i}, p_{i+1}\right]$ and $\left[p_{a+k-1}, p_{i+k}\right]$, then, clearly, $G_{i}$ contains the vertices of no diameter of $P$. Thus, the arcs $G_{i+t(k-1)}$, where $t=1,2, \ldots, 2 k+\left\lceil\frac{2 k}{m-1}\right\rceil$, form a $k$-fold Borsuk-covering of $\operatorname{bd} P$.

REMARK 5.3. It is proven in [4] that in any angled normed plane there is a complete set of Borsuk number two. In other words, for a normed plane, the result in [1] about the Borsuk numbers of sets in the Euclidean plane holds in the same form if, and only if the plane is not angled. According to our results, the same can be observed about the multiple Borsuk numbers of sets.

REMARK 5.4. In any angled normed plane, there is a Borsuk covering of a set of unique completion, satisfying the supporting line property (see page 2), that cannot be completed to a Borsuk covering of its completion.

PROOF. If the norm is a parallelogram norm, the remark trivially follows. Hence, we may assume that the unit disk $\mathbf{B}$ is not a parallelogram, and that 
its boundary contains $[x, y] \cup[y, z]$ and $[-x,-y] \cup[-y,-z]$. Without loss of generality, we may assume that the lines, containing $[x, y]$ and $[y, z]$, intersect $\mathbf{B}$ in $[x, y]$ and $[y, z]$, respectively.

Let $C$ be the truncation of $\mathbf{B}$ with a line connecting the relative interior points $w_{1}$ and $w_{2}$ of $[x, y]$ and $[y, z]$, respectively. Clearly, the unique completion of $C$ is $\mathbf{B}$, and $C$ satisfies the supporting line property. Let $w$ be the midpoint of $\left[w_{1}, w_{2}\right]$. Let $u_{1}$ and $u_{2}$ be relative interior points of $[-x,-y]$ and $[-y,-z]$, respectively (cf. Figure 3 ). Then the shorter $\operatorname{arcs}$ of bd $C$ connecting $w$ to $u_{1}, u_{1}$ to $u_{2}$, and $u_{2}$ to $w$, is a Borsuk covering of bd $C$. On the other hand, $y$ cannot be added to any of these arcs, which yields that this covering cannot be extended to any Borsuk covering of $\mathbf{B}$.

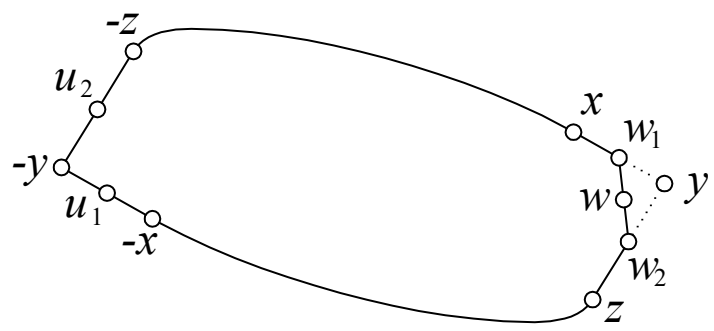

Fig. 3. A Borsuk covering may not be extended in an angled plane

Note that if $\mathbf{B}$ is a parallelogram, then the only complete sets of unit diameter are the translates of $\mathbf{B}$ (cf. [22], [17] or [13]).

REMARK 5.5. Let $S$ be a compact set with $a(S)=3$ in the normed plane where $\mathbf{B}$ is a parallelogram. Then $a_{k}(S)=3 k$ for every $k$.

Proof. Without loss of generality, let $\mathbf{B}$ be the unique completion of $S$. By the supporting line property, $S$ contains at least two consecutive vertices of $\mathbf{B}$. Furthermore, since $\mathbf{B}$ is the unique completion, $S$ contains a point of the opposite side of $\mathbf{B}$. Thus, $S$ contains three points at pairwise normed distances equal to diam $S$, which yields $a_{k}(S) \geq 3 k$. By sub-additivity, we have $a_{k}(S) \leq 3 k$, and the assertion readily follows.

Acknowledgment. Both authors acknowledge the support of the János Bolyai Research Scholarship of the Hungarian Academy of Sciences. The second named author thanks also the National Research, Development and Innovation Office, NKFIH Grants PD104744 and K119670.

\section{REFERENCES}

[1] Boltyanski, V. G., On the partition of plane figures into pieces of smaller diameters (in Russian), Colloq. Math., 21(1970), 253-263. 
[2] Boltyanski V. G. and Gohberg, I. C., The Decomposition of Figures into Smaller Parts, translated from Russian, The University of Chicago Press, Chicago, 1980.

[3] Boltyanski, V., Martini, H. and Soltan, P. S., Excursions into Combinatorial Geometry, Springer-Verlag, Berlin Heidelberg, 1997.

[4] Boltyanski, V. G. and Soltan, V., Borsuk's problem (in Russian), Mat. Zametki 22(1977), 621-631.

[5] Borsuk, K., Drei Sätze über die $n$-dimensionale eukildische Sphäre, Fundamenta Math., 20(1933), 177-190.

[6] Eggleston, H. G., Covering a three-dimensional set with sets of smaller diameter, J. London Math. Soc., 30(1955), 11-24.

[7] Eggleston, H. G., Sets of constant width in finite dimensional Banach spaces, Israel J. Math., 3(1965), 163-172.

[8] Grünbaum, B., Borsuk's partition conjecture in Minkowski planes, Bull. Res. Council Israel, F1, N1,(1957), 25-30.

[9] Grünbaum, B., A simple proof of Borsuk's conjecture in three dimensions, Proc. Cambridge Philos. Soc., 53(1957), 776-778.

[10] Hammer, P. C., Convex curves of constant Minkowski breadth, Proc. Symp. Pure Math., 7, Amer. Math. Soc., Providence RI (1963), 291-304.

[11] Heppes, A., On the partitioning of a three-dimensional point set into sets of smaller diameter (Hungarian), Magyar Tud. Akad. Mat. Fiz. Oszt. Közl., 7(1957), 413-416.

[12] Hujter, M. and LÁngi, Z., On the multiple Borsuk numbers of sets, Israel J. Math., 199(2014), 219-239.

[13] Joós, A. and LÁNGI, Z., On the relative distances of seven points in a plane convex body, J. Geom., 87(2007), 83-95.

[14] Kahn, J. and Kalai, G., A counterexample to Borsuk's conjecture, Bull. Amer. Math. Soc., 29(1993), 60-62.

[15] Lassak, M., On relatively equilateral polygons inscribed in a convex body, Publ. Math. Debrecen 65(2004), 133-148.

[16] Moreno, J.P., Porosity and unique completion in strictly convex spaces, Math. Z. 267(1-2) (2011), 173-184.

[17] NASzóDI, M. and VISY, B., Sets with a unique extension to a set of constant width, Discrete geometry, Monogr. Textbooks Pure Appl. Math., 253, Dekker, New York, 2003, 373-380.

[18] Raigorodskit, A.M., Around Borsuk's hypothesis, Journal of Mathematical Sciences 154(2008), no. 4, 604-623 (English).

[19] Sallee, G.T., The maximal set of constant width in a lattice, Pacific J. Math. 28(1969), 669-674.

[20] Stahl, S., $n$-tuple colorings and associated graphs, J. Combin. Theory Ser. B 20(1976), 185-203.

[21] Thompson, A.C., Minkowski Geometry, Encyclopedia of Mathematics and its Applications 63, Cambridge University Press, Cambridge, 1996.

[22] Yost, D., Irreducible convex sets, Mathematika, 38(1991), no. 1, 134-155. 